APS/123-QED

\title{
Force transmission in a packing of pentagonal particles
}

\author{
Emilien Azéma, Farhang Radjaï, Robert Peyroux \\ LMGC, CNRS - Université Montpellier II, Place Eugène Bataillon, 34095 Montpellier cedex 05, France* \\ Gilles Saussine \\ Innovation and Research Departement of SNCF, 45 rue de Londres, 75379 PARIS Cedex 08
}

(Dated: August 14, 2018)

\begin{abstract}
We perform a detailed analysis of the contact force network in a dense confined packing of pentagonal particles simulated by means of the contact dynamics method. The effect of particle shape is evidenced by comparing the data from pentagon packing and from a packing with identical characteristics except for the circular shape of the particles. A counterintuitive finding of this work is that, under steady shearing, the pentagon packing develops a lower structural anisotropy than the disk packing. We show that this weakness is compensated by a higher force anisotropy, leading to enhanced shear strength of the pentagon packing. We revisit "strong" and "weak" force networks in the pentagon packing, but our simulation data provide also evidence for a large class of "very weak" forces carried mainly by vertex-to-edge contacts. The strong force chains are mostly composed of edge-to-edge contacts with a marked zig-zag aspect and a decreasing exponential probability distribution as in a disk packing.
\end{abstract}

PACS numbers:

\section{INTRODUCTION}

Among singular features of granular media, force transmission has received particular interest during the last decade. The contact forces in model granular media, as observed by experiments and numerical simulations, are highly inhomogeneous and their probability density func-

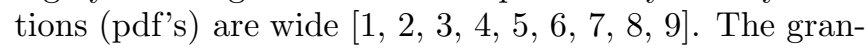
ular texture is generically anisotropic in two respects: 1) The contact normal directions are not random; 2) The force average as a function of contact normal direction is not uniform. The corresponding fabric and force anisotropies in shear are responsible for mechanical strength at the scale of the packing [10, 11, 12, 13]. Another interesting aspect, first analyzed in Ref. [10] is the fact that the forces organize themselves in two distinct classes which contribute differently to fabric anisotropy, shear stress, and dissipation. In particular, the shear stress is fully transmitted via a "strong" contact network, materialized by force "chains". The stability is ensured by the antagonist role of "weak" contacts which prop strong force chains 10, 14].

The force transmission properties have been for the most part investigated in the case of granular media composed of isometric (circular or spheric) particles. However, in various fields of science and engineering, the grains are seldom so "perfect". For example, elongated and platy shapes are encountered in biomaterials or pharmaceutical applications. Such shapes have unequal dimensions and induce thus a degree of anisotropy in the bulk behavior in addition to fabric and force anisotropies [15, 16, 17, 18]. On the other hand, granular geo-

*Electronic address: azema@lmgc.univ-montp2.fr materials are often composed of angular particles with plane faces as polyhedra. While rounded particles enhance flowability, angular shape is susceptible to enhance the shear strength, a factor of vital importance to civilengineering applications [18, 19, 20]. The railway ballast is a well-known case where particle shape must be optimized to avoid excessive differential settlement under vertical loading 21, 22, 23]. In such circumstances, the analysis of force transmission is a key to improve performance.

In dealing with effects of particle shape, the issue is that a general quantitative description of particle morphology requires various shape parameters. For regular polygons in $2 \mathrm{D}$, for instance, the only shape parameter is the number of sides (besides the diameter) whereas for irregular polygons more information is needed about the positions of the vertices in a reference system attached to the particle. In soil mechanics, angularity and roundedness are among basic parameters used to describe particle shapes 24]. As far as force transmission is concerned, at least two parameters seem to be most relevant: 1) shape anisotropy (anisometry), which contributes to the anisotropy of stress transmission [15];2) facettedness, which allows for extended (face to face, edge to face and edge to edge) contacts between particles leading possibly to the formation of columnar structures within a granular assembly.

In this paper, we consider one of the simplest possible shapes, namely regular pentagons. Among regular polygons, the pentagon has the lowest number of sides, corresponding to the least roundedness in this category, without the pathological space-filling properties of triangles and squares. We seek to isolate the effect of edgeto-edge contacts on force transmission by comparing the data with a packing of circular particles that, apart from the particle shape, is identical in all respects (prepara- 
tion, friction coefficients, particle size distribution) to the pentagon packing. Both packings are subjected to biaxial compression simulated by means of the contact dynamics method. The presence of edge-to-edge contacts affects both quantitatively and qualitatively the microstructure and the overall behavior during shear. These contacts do not transmit torques, but they are able to accommodate force lines that are usually unsustainable in packings of disks.

This paper is organized as follows. We first present in Section [the numerical procedures and a brief technical introduction to the detection and treatment of edge-toedge contacts in the framework of the contact dynamics method. In Section [II] we compare stress-strain and volume-change characteristics. Then, In Sections IV and V we analyze the texture and force transmission features. In Section VI we focus on the pentagon packing and we analyze the structure of force networks with vertex-to-edge and edge-to-edge contacts. The main results are summarized and discussed in Section VII.

\section{NUMERICAL PROCEDURES}

The simulations were carried out by means of the contact dynamics (CD) method [25, 26]. The CD method is based on implicit time integration of the equations of motion and a nonsmooth formulation of mutual exclusion and dry friction between particles. This method requires no elastic repulsive potential and no smoothing of the Coulomb friction law for the determination of forces. For this reason, the simulations can be performed with large time steps compared to molecular dynamics simulations. We used the platform LMGC90 which is a multipurpose software developed in Montpellier, capable of modeling a collection of deformable or undeformable particles of various shapes [27].

\section{A. Contact dynamics for polygons}

The particles are rigid polygons exerting normal and shear forces, $f_{n}$ and $f_{t}$, respectively, on each other. We attribute a positive sign to compressive normal forces. The relative normal velocity $u_{n}$ between two particles in contact is counted positive when they move away from each other. Then, the condition of geometrical contact between two particles is expressed by the following mutually exclusive alternatives:

$$
\begin{array}{ll}
f_{n} \geqslant 0 & u_{n}=0 \\
f_{n}=0 & u_{n}>0 .
\end{array}
$$

In the same way, the Coulomb friction law involves three mutually exclusive conditions:

$$
\begin{array}{cc}
f_{t}=-\mu f_{n} & u_{t}>0 \\
-\mu f_{n} \leqslant f_{t} \leqslant \mu f_{n} & u_{t}=0 \\
f_{t}=\mu f_{n} & u_{t}<0
\end{array}
$$

where $u_{t}$ is the sliding velocity at the contact and $\mu$ is the friction coefficient. The unknown variables are particle velocities and contact forces. These are calculated at each time step by taking into account the conservation of momenta, the constraints expressed by (11) and (2), and the dissipation of kinetic energy during inelastic collisions between particles (ref). We use an iterative research algorithm based on a nonlinear Gauss-Seidel scheme. The uniqueness is not guaranteed for perfectly rigid particles in absolute terms. However, by initializing each step of calculation with the forces calculated in the preceding step, the set of admissible solutions shrinks to fluctuations which are basically below the numerical solution. Let us note that in molecular dynamics simulations, this "force history" is encoded by construction in the particle positions.

The research algorithm is applied to a set of potential contacts, identified or updated in each step. The contact detection between two bodies consists in looking for the overlaps of the portions of space they occupy. The treatment of the mechanical interaction requires additionally the identification of a common tangent plane (a line in 2D). Of course, contact may take place through a larger contact zone than a single point. Several algorithms exist for overlap determination between convex polygons 21, 27]. In 2D simulations of the present paper, the detection of contact between two convex polygonal bodies was implemented through the so-called "shadow overlap method" devised by Moreau [21, 27], with reliability and robustness tested in several years of previous applications to various states of granular materials [18, 28, 29].

In detection of contacts between two polygons, two situations arise: 1) If a single corner is found crossing an edge of the partner polygon, the direction of this edge is viewed as the tangent direction. By orthogonally projecting the intruding vertex onto the edge, one determines the penetration depth, while the nominal contact point is chosen at the center of this distance. Below, we will refer to this vertex-to-edge contact as "simple" contact. 2) In case of double intrusion, the common tangent line is fixed from as a mean between the two overlapping edges and a segment of this line is identified as the contact segment. The impenetrability between two particles at such an edge-to-edge contact is ensured by applying the contact laws (1) and (2) to only two points of the contact segment (Fig. 11). For this reason, we refer below to edge-to-edge contacts as "double" contacts. In practice, two forces are calculated at each double contact, but only their resultant and application point are material. In this respect, the choice of the two points representing a double contact does not affect the dynamics of the system.

\section{B. Numerical samples}

We generated two numerical samples. The first sample, denoted S1, is composed of 14400 regular pentagons of 

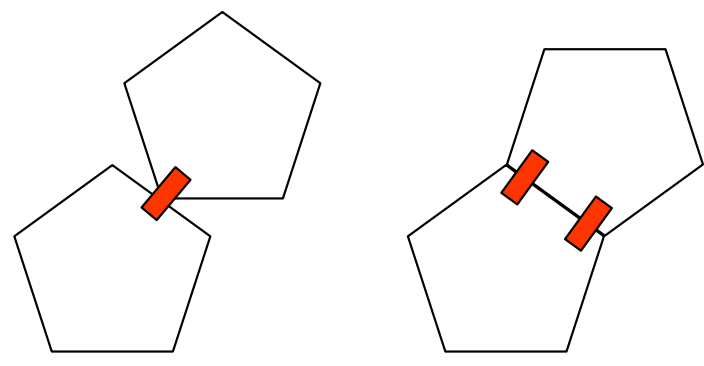

FIG. 1: Representation of simple (vertex-to-edge) and double (edge-to-edge) contacts between two pentagons.

three different diameters: $50 \%$ of diameter $2.5 \mathrm{~cm}, 34 \%$ of diameter $3.75 \mathrm{~cm}$ and $16 \%$ of diameter $5 \mathrm{~cm}$. The second sample, denoted S2, is composed of 10000 discs with the same polydispersity. Both samples were prepared according to the same protocol. A dense packing was first constructed following simple geometrical rules [30] and then compressed isotropically under a constant stress $\sigma_{0}=10^{4}$ $\mathrm{Pa}$ applied onto the right and top walls. The gravity was set to zero in order to avoid force gradients in the samples. The coefficient of friction was set to 0.4 between grains and to 0 with the walls. At equilibrium, both numerical samples were in isotropic stress state. The solid fraction was $\phi_{0}=0.80$ for S1 and $\phi_{0}=0.82$ for S2. The aspect ratio was $h / l \approx 2$, where $h$ and $l$ are the height and width of the sample, respectively. Figure 2 displays snapshots of the two packings at the end of isotropic compaction.

The isotropic samples were subjected to vertical compression by downward displacement of the top wall at a constant velocity of $1 \mathrm{~cm} / \mathrm{s}$ for a constant confining stress $\sigma_{0}$ acting on the lateral walls. The simulations were run up to a total cumulative vertical strain of 0.2 with a time step of $5 \cdot 10^{-4} \mathrm{~s}$. The CPU time was $7.10^{-4}$ $\mathrm{s}$ and $5.10^{-4} \mathrm{~s}$ per particle and per time step on a G5 Apple computer. Since we are interested in quasistatic behavior, the shear rate should be such that the kinetic energy supplied by shearing is negligible compared to the static pressure. This can be formulated in terms of an "inertia parameter" I [31] defined by

$$
I=\dot{\varepsilon} \sqrt{\frac{m}{p}}
$$

where $\dot{\varepsilon}=\dot{y} / y$ is the strain rate, $m$ is the total mass, and $p$ is the average pressure. The quasistatic limit is characterized by the condition $I \ll 1$. In our biaxial simulations, $I$ was below $10^{-3}$.

\section{STRENGTH AND DILATANCY}

In this section, we compare the stress-strain and volume-change behavior between the packings of poly-
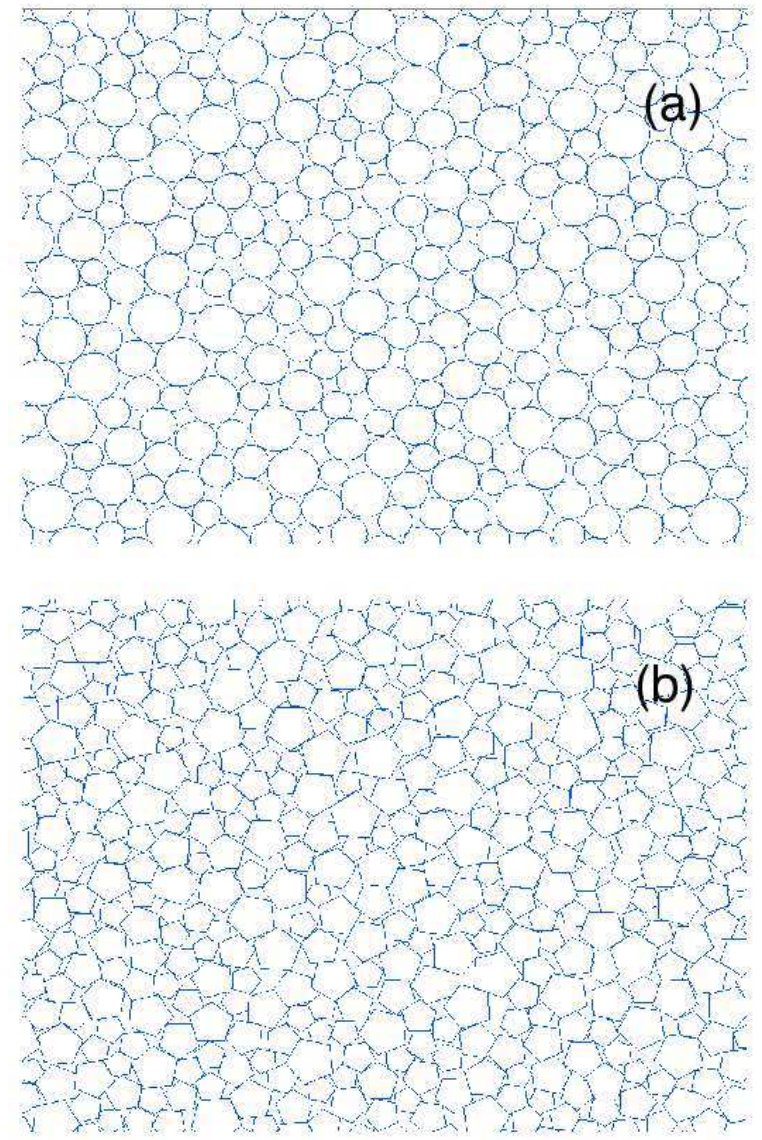

FIG. 2: Snapshots of a portion of the samples S2 (a) and S1 (b) composed of circular and pentagonal particles, respectively.

gons (sample S1) and disks (sample S2). For the calculation of the stress tensor, we consider the "tensorial moment" $M^{i}$ of each particle i defined by [14, 32]:

$$
M_{\alpha \beta}^{i}=\sum_{c \in i} f_{\alpha}^{c} r_{\beta}^{c},
$$

where $f_{\alpha}^{c}$ is the $\alpha$ component of the force exerted on particle $\mathrm{i}$ at the contact $\mathrm{c}, r_{\beta}^{c}$ is the $\beta$ component of the position vector of the same contact $\mathrm{c}$, and the summation is runs over all contacts c of neighboring particles with the particle i (noted briefly by $c \in i$ ). It can be shown that the tensorial moment of a collection of rigid particles is the sum of the tensorial moments of individual particles. The stress tensor $\boldsymbol{\sigma}$ for a packing of volume $V$ is simply given by [14, 32$]$ :

$$
\boldsymbol{\sigma}=\frac{1}{V} \sum_{i \in V} \boldsymbol{M}^{i}=\frac{1}{V} \sum_{c \in V} f_{\alpha}^{c} \ell_{\beta}^{c},
$$

where $\boldsymbol{\ell}^{c}$ is the intercenter vector joining the centers of the two touching particles at the contact $c$. Remark that the first summation runs over all particles whereas the second summation involves all contacts in the volume 


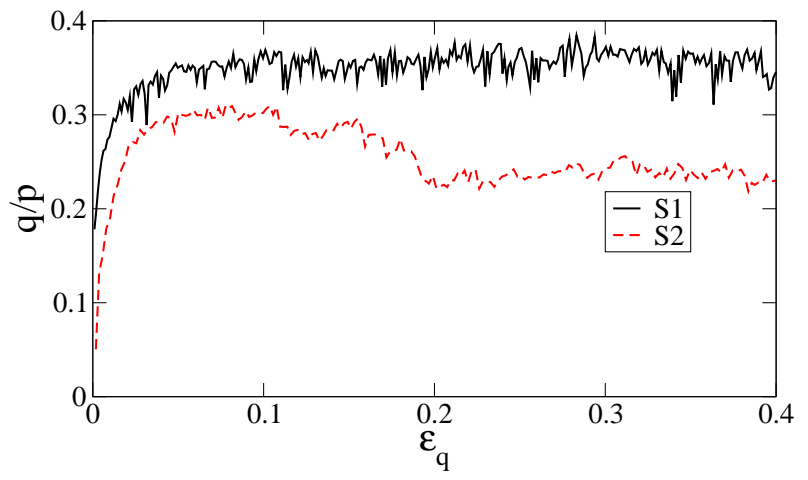

FIG. 3: Normalized shear stress $q / p$ as a function of cumulative shear strain $\varepsilon_{q}$ for the samples S1 and S2.

$V$, with each contact appearing only once. We extract the mean stress $p=\left(\sigma_{1}+\sigma_{2}\right) / 2$, and the stress deviator $q=\left(\sigma_{1}-\sigma_{2}\right) / 2$, where $\sigma_{1}$ and $\sigma_{2}$ are the principal stresses. The major principal direction during vertical compression is vertical.

The strain parameters are the cumulative vertical, horizontal and volumetric strains $\varepsilon_{1}, \varepsilon_{2}$ and $\varepsilon_{p}$, respectively. By definition, we have

$$
\varepsilon_{1}=\int \frac{d h}{h}=\ln \left(1+\frac{\Delta h}{h_{0}}\right),
$$

where $h_{0}$ is the initial height and $\Delta h=h_{0}-h$ is the total downward displacement, and

$$
\varepsilon_{p}=\int \frac{d V}{V}=\ln \left(1+\frac{\Delta V}{V_{0}}\right)
$$

where $V_{0}$ is the initial volume and $\Delta V=V-V_{0}$ is the cumulative volume change.

Figure 3 shows the normalized shear stress $q / p$ for the samples S1 and S2 as a function of shear strain $\varepsilon_{q} \equiv \varepsilon_{1}-$ $\varepsilon_{2}$. For $\mathrm{S} 2$, we observe a classical behavior characterized by a hardening behavior followed by (slight) softening and a stress plateau corresponding to the residual state of soil mechanics 24]. For S1, we observe no marked stress peak. The residual stress is higher for polygons $(\simeq 0.35)$ than for disks $(\simeq 0.28)$. This means that the polygon packing has a higher angle of internal friction $\varphi$ defined by

$$
\sin \varphi=\frac{q}{p}
$$

Figure 4 displays the cumulative volumetric strain $\varepsilon_{p}$ for polygons and disks as a function of $\varepsilon_{q}$. Both samples dilate and tend to isochoric deformation at large strains. It is remarkable that the polygon packing S1 initially dilates less than the disk packing S2. This behavior is reversed at larger strains with a crossover occurring after the peak state. Notice that the solid fraction is initially lower in S1 (0.80) than in S2 (0.82). This is because it is more difficult to obtain a compact packing with polygonal shapes by isotropic compression as

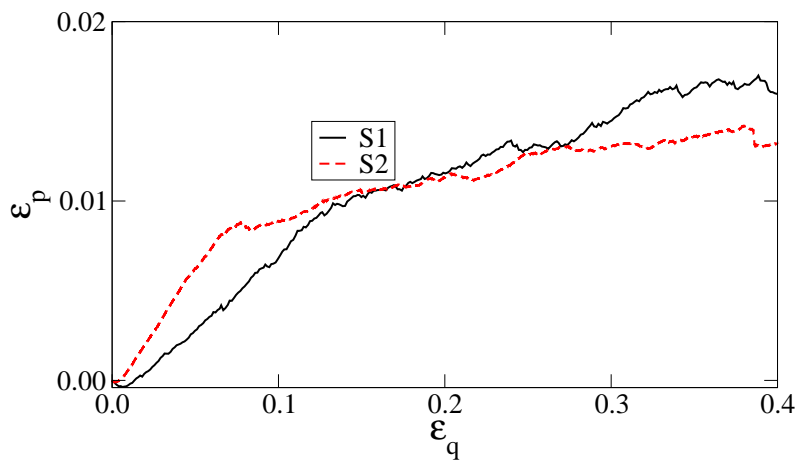

FIG. 4: Cumulative volumetric strain $\varepsilon_{p}$ as a function of cumulative shear strain $\varepsilon_{q}$ for the samples S1 and S2.

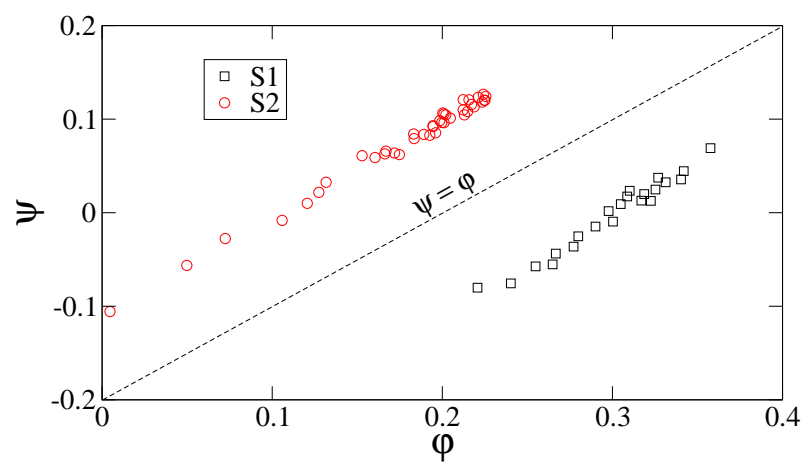

FIG. 5: Stress-dilatancy relation between dilatancy angle $\psi$ and internal angle of friction $\varphi$ for the samples S1 and S2.

a result of enhanced steric effects compared to disks. In other words, angular particles can form larger pores compared to rounded particles. The volumetric deformation can also be expressed in terms of the so-called "dilatancy angle" $\psi$ defined by [33]

$$
\sin \psi=\frac{\varepsilon_{p}}{\varepsilon_{q}}
$$

The cumulative angle of dilatancy, i.e. during shear up to the residual state, is only slightly higher for the polygon packing than the disk packing.

The plot of $\psi$ as a function of $\varphi$, i.e. the so-called stress-dilatancy diagram, is shown in Fig. [5 for polygons and disks [33]. Remarkably, both plots are parallel to the line $\varphi=\psi$ with an offset $\varphi_{0}$ :

$$
\varphi \simeq \varphi_{0}+\psi
$$

The offset $\varphi_{0}$ is the friction angle at zero dilatancy. We have $\varphi_{0} \simeq 0.12$ for disks and $\varphi_{0} \simeq 0.3$ for polygons. This observation is in agreement with the arguments of Taylor [33, 34] based on energy balance and recently revisited also in the case of cohesive granular media [35]. The higher level of $\varphi$ for the polygon packing reflects the organization of the microstructure and the features of force transmission for each particle shape. This point is considered in more detail in the following section. 


\section{GRANULAR TEXTURE}

The granular texture, i.e. the organization of the particles and their contacts in space, is basically controlled by steric exclusions between the particles and force balance conditions [36]. The texture can be described in terms of various statistical descriptors pertaining to the forcebearing network of particles. At the lowest order, the compactness of the structure can be described in terms of both the solid fraction $\rho$ and the coordination number $z$. The connectivity of the network can further be characterized by the fraction $P(c)$ of particles having exactly $c$ contact neighbors. These are scalar parameters or functions. At higher orders, the anisotropy of the texture is described by different "fabric tensors". We consider here these geometrical descriptors in order to identify the signature of particle shape.

\section{A. Connectivity}

The connectivity of the particles by force-bearing contacts is described at the lowest order by the average number $z$ of contact neighbors per particle. The particles with no force-bearing contact are thus removed from the statistics. Note also that each double (edge-to-edge) contact for the polygons is counted once although double contacts are treated as two point contacts belonging to the contact segment (see section III). Fig. 6a displays the evolution of $z$ for the pentagon packing (S1) and the disk packing (S2) as a function of $\varepsilon_{q}$. The coordination number evolves to a steady-state value in both samples that is higher for $\mathrm{S} 2(\simeq 3.85)$ than for $\mathrm{S} 1(\simeq 3.75)$. The difference is, however, much less important than in the initial configuration $(\simeq 3.95$ for $\mathrm{S} 2$ compared to $\simeq 3.20$ for S1) prepared by means of isotropic compaction.

It is also interesting to compare the two samples in terms of "contact lifetimes". Let us consider a reference configuration, e.g. the initial state of each sample. We follow the history of each contact listed in this state. In particular, we define $\gamma$ as the fraction of persistent contacts of the initial list. During deformation, $\gamma$ declines from 1 to 0 as an increasing number of initial contacts are lost due to particle rearrangements. Fig. 60 shows gamma as a function of $\varepsilon_{q}$ for $\mathrm{S} 1$ and $\mathrm{S} 2$. We see that, following a rapid initial falloff, $\gamma$ decreases slowly in both samples but the rate of contact loss is globally higher for polygons than disks. We remark that even at $\varepsilon_{q}=0.4$, the contact list is renewed by only $50 \%$.

The connectivity $P(c)$ of the particles is plotted in Fig. 7 for $\mathrm{S} 1$ and $\mathrm{S} 2$ at $\varepsilon_{q}=0.3$. Interestingly, the two plots are nearly identical with a peak for $c=4$. In both samples, the fraction of particles with 5 contacts is larger than that with 3 contacts. This shows that the connectivity does not reflect the difference in texture between the two packings although a qualitative difference exists as we shall see below by considering fabric anisotropy and force transmission.
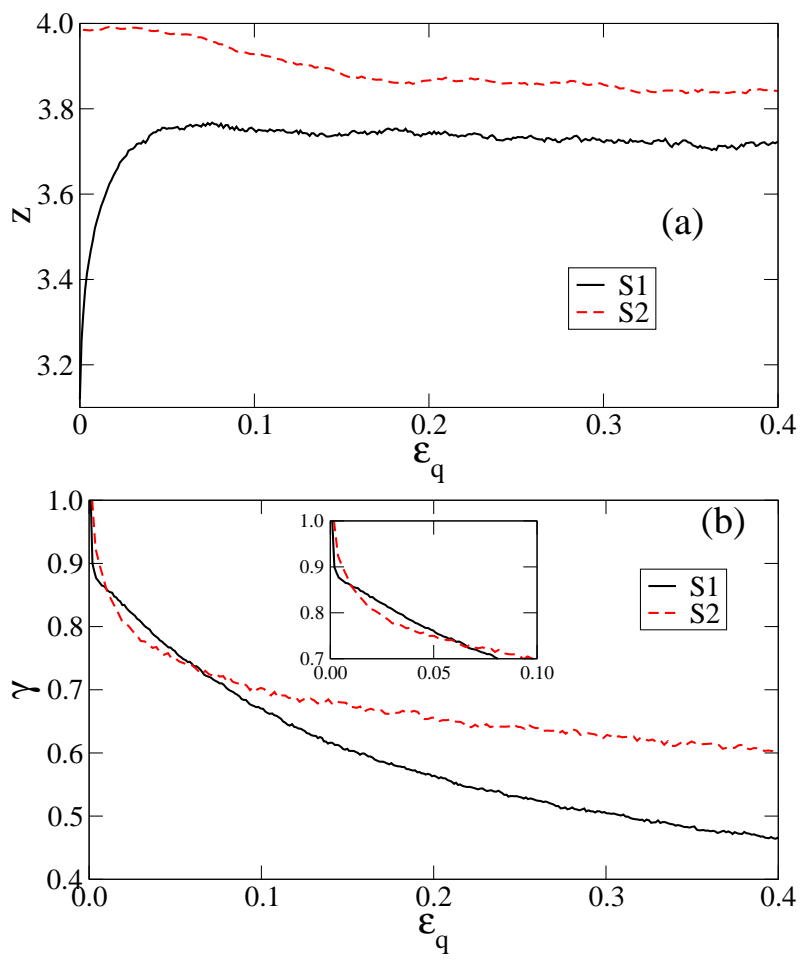

FIG. 6: The coordination number $z$ (a) and the fraction $\gamma$ of persistent contacts (b) as a function of cumulative shear strain $\varepsilon_{q}$ for the samples S1 and S2.

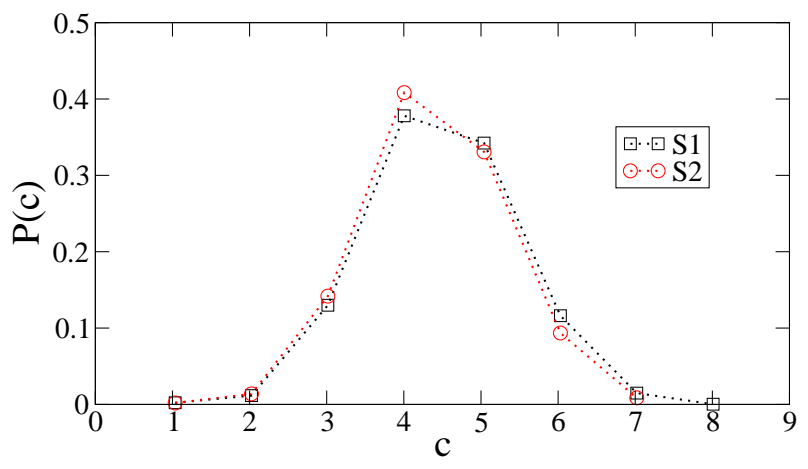

FIG. 7: Connectivity diagram for the samples S1 and S2 expressing the fraction $P(c)$ of particles with exactly $c$ contacts in the residual state.

\section{B. Fabric anisotropy}

The shear strength of dry granular materials is generally attributed to the buildup of an anisotropic structure during shear due to friction between the particles and as a result of steric effects depending on particle shape [37, 38, 39]. Several methods have been used to quantify the fabric (structural) anisotropy of granular materials [13, 40, 41]. A common approach is to consider the probability distribution $P(\boldsymbol{n})$ of the contact normals $\boldsymbol{n}$ which are generically nonuniform. In two dimensions, the unit vector $\boldsymbol{n}$ is described by a single angle $\theta$, the orientation 


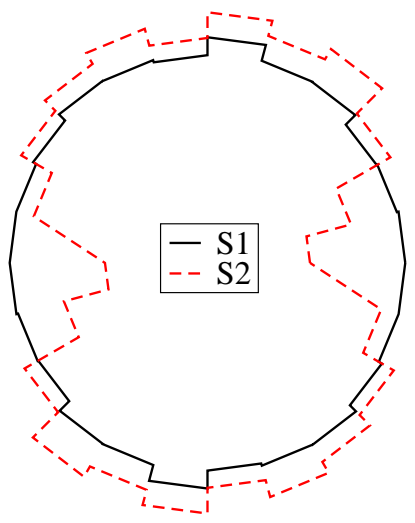

FIG. 8: Polar representation of the probability density function $P_{\theta}$ of the contact normal directions $\theta$ for the samples S1 and $\mathrm{S} 2$ in the residual state.

of the contact normal. The probability density function $P_{\theta}(\theta)$ of contact normals provides a detailed statistical information about the fabric. It is $\pi$-periodic in the absence of an intrinsic polarity for $\boldsymbol{n}$.

Most structural information is generally condensed in the second moment of $P_{\theta}$, called fabric tensor [40]:

$$
F_{\alpha \beta}=\frac{1}{\pi} \int_{0}^{\pi} n_{\alpha}(\theta) n_{\beta}(\theta) P_{\theta}(\theta) d \theta \equiv \frac{1}{N_{c}} \sum_{c \in V} n_{\alpha}^{c} n_{\beta}^{c},
$$

where $\alpha$ and $\beta$ design the components in a reference frame and $N_{c}$ is the total number of contacts in the control volume $V$. By definition, $\operatorname{tr}(\boldsymbol{F})=1$. The anisotropy of the contact network is given the difference between the principal values $F_{1}$ and $F_{2}$. We define the fabric anisotropy $a$ by

$$
a=2\left(F_{1}-F_{2}\right) .
$$

For fix coordinates, with the $\mathrm{x}$-axis pointing along $\theta^{\prime}$, we define also a "signed anisotropy" $a^{\prime}$ by

$$
a^{\prime}=2\left(F_{1}-F_{2}\right) \cos 2\left(\theta_{F}-\theta^{\prime}\right),
$$

where $\theta_{F}$ is the major principal direction of the fabric tensor. For $\theta^{\prime}=\theta_{F}$, we have $a^{\prime}=a$. The signed anisotropy corresponds to the second term of the Fourier expansion of $P_{\theta}(\theta)$ and it is useful whenever the direction of anisotropy is not constant.

Figure 8 displays a polar representation of $P_{\theta}(\theta)$ for the samples $\mathrm{S} 1$ and $\mathrm{S} 2$ at $\varepsilon_{q}=0.3$. We observe a nearly isotropic distribution for the pentagon packing in spite of shearing whereas the disk packing is markedly anisotropic. This is a surprising observation in view of the higher shear strength of the pentagon packing (Fig. 3). It is also counterintuitive as one expects that double contacts should allow a polygon packing to build more easily an anisotropic structure.

The evolution of $a^{\prime}$ is shown in Fig. 9 as a function of $\varepsilon_{q}$ for S1 and S2. The privileged direction of the contacts, corresponding to $\theta_{F}$, is vertical in both packings.

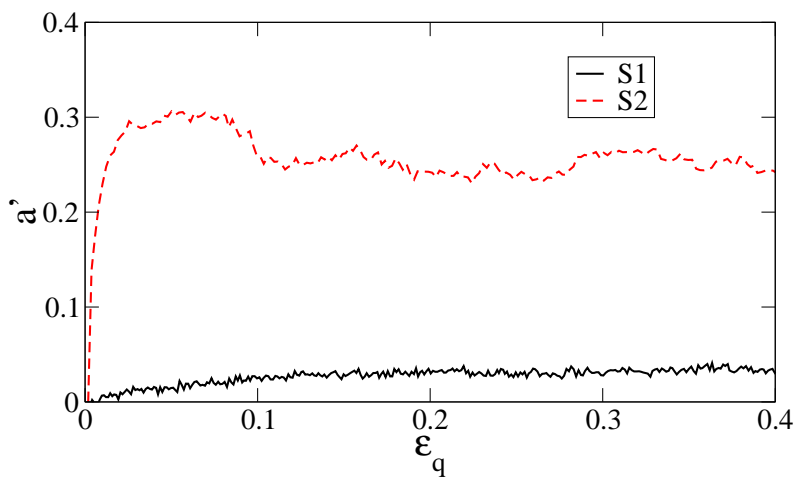

FIG. 9: Evolution of the anisotropy $a^{\prime}$ with cumulative shear strain $\varepsilon_{q}$ for the samples S1 and S2.

In both cases, $a^{\prime}$ increases from 0 (as a result of the initial isotropic compression) to its largest value in the residual state. The anisotropy stays quite weak in the pentagon packing whereas the disk packing is marked by a much larger anisotropy, increasing to $\simeq 0.3$ and then relaxing to a slightly lower value in the residual state. As we shall see below, the low anisotropy of the pentagon packing results from a particular organization of the force network in correlation with the orientations of simple and double contacts in the packing (section VI). We will also show that the large shear strength of the pentagon packing is a consequence of a strong force anisotropy in this packing (see next section).

\section{FORCE TRANSMISSION}

In this section, we analyze the anisotropy and inhomogeneity of force networks in the packings of pentagons and disks. This leads us to consider the contributions of force and texture anisotropies to average shear stresses.

\section{A. Force anisotropy}

The angular distribution of contact forces in a granular packing can be represented by the average force $\langle\boldsymbol{f}\rangle(\boldsymbol{n})$ as a function of the contact normal direction $\boldsymbol{n}$. We distinguish the average normal force $\left\langle f_{n}\right\rangle$ from the average tangential force $\left\langle f_{t}\right\rangle$ formally defined by [13]

$$
\left\{\begin{array}{l}
\left\langle f_{n}\right\rangle(\theta)=\frac{1}{N_{c}(\theta)} \sum_{c \in \mathcal{S}(\theta)} f_{n}^{c}, \\
\left\langle f_{t}\right\rangle(\theta)=\frac{1}{N_{c}(\theta)} \sum_{c \in \mathcal{S}(\theta)} f_{t}^{c},
\end{array}\right.
$$

where $f_{n}^{c}$ and $f_{t}^{c}$ are the normal and tangential forces, respectively, acting at the contact $c$ (according to a sign convention attributing positive values to the normal forces), $\mathcal{S}(\theta)$ is the set of contacts with direction $\theta \in[\theta-\Delta \theta / 2, \theta+\Delta \theta / 2]$ for angle increments $\Delta \theta$, and $N_{c}(\theta)$ is the number of contacts in $\mathcal{S}(\theta)$. 


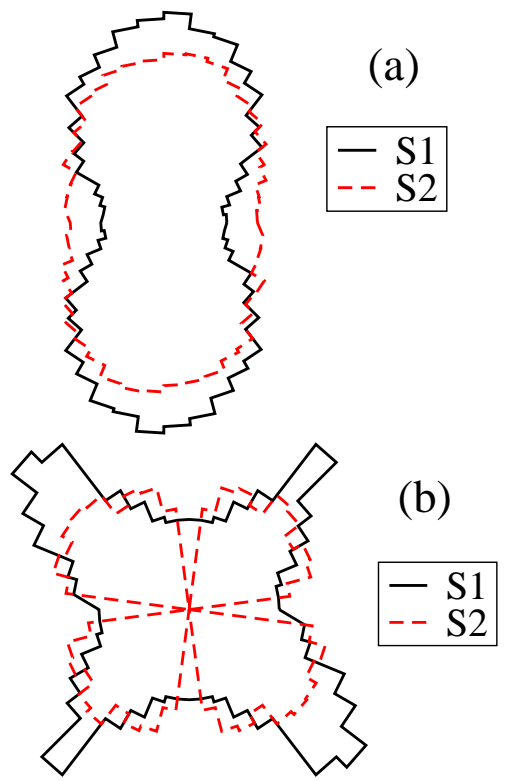

FIG. 10: Polar representation of the angle-averaged normal (a) and tangential (b) forces $\left\langle f_{n}\right\rangle(\theta)$ and $\left\langle f_{t}\right\rangle(\theta)$ for the samples $\mathrm{S} 1$ and $\mathrm{S} 2$ in the residual state.

By definition, the two functions $\left\langle f_{n}\right\rangle$ and $\left\langle f_{t}\right\rangle$ are $\pi$ periodic. After sufficiently long monotonous shearing, these functions can be approximated by their Fourier expansions truncated beyond the second term [13, 39]:

$$
\left\{\begin{array}{l}
\left\langle f_{n}\right\rangle(\theta)=\langle f\rangle\left\{1+a_{n} \cos 2\left(\theta-\theta_{n}\right)\right\} \\
\left\langle f_{t}\right\rangle(\theta)=\langle f\rangle a_{t} \sin 2\left(\theta-\theta_{t}\right)
\end{array}\right.
$$

where $\langle f\rangle$ is the average force, $a_{n}$ and $a_{t}$ represent the anisotropies of the normal and tangential forces, respectively, and $\theta_{n}$ and $\theta_{t}$ are their privileged directions.

In Fig. 10, the functions $\left\langle f_{n}\right\rangle(\theta)$ and $\left\langle f_{t}\right\rangle(\theta)$ are displayed in polar coordinates at $\varepsilon_{q}=0.3$. The pentagon and disk packings show pronounced force anisotropy with a stronger anisotropy in the case of pentagons both for normal and tangential forces. These plots can be fitted by harmonic functions [Eq. (15)] in order to estimate the force anisotropies $a_{n}$ and $a_{t}$. However, it is more convenient to estimate the anisotropies through the following "force tensors":

$$
\left\{\begin{array}{l}
H_{\alpha \beta}^{(n)}=\int_{0}^{\pi}\left\langle f_{n}\right\rangle(\theta) n_{\alpha} n_{\beta} d \theta, \\
H_{\alpha \beta}^{(t)}=\int_{0}^{\pi}\left\langle f_{t}\right\rangle(\theta) n_{\alpha} n_{\beta} d \theta .
\end{array}\right.
$$

It is easy to see that $\operatorname{tr}\left(\boldsymbol{H}^{(n)}\right)=\operatorname{tr}\left(\boldsymbol{H}^{(t)}\right)=\langle f\rangle$, and by identification with (15) we have

$$
\left\{\begin{array}{l}
a_{n}=2 \frac{H_{1}^{(n)}-H_{2}^{(n)}}{H_{1}^{(n)}+H_{2}^{(n)}}, \\
a_{t}=2 \frac{H_{1}^{(t)}-H_{2}^{(t)}}{H_{1}^{(t)}+H_{2}^{(t)}},
\end{array}\right.
$$

where the subscripts 1 and 2 refer to the principal values of the tensors.
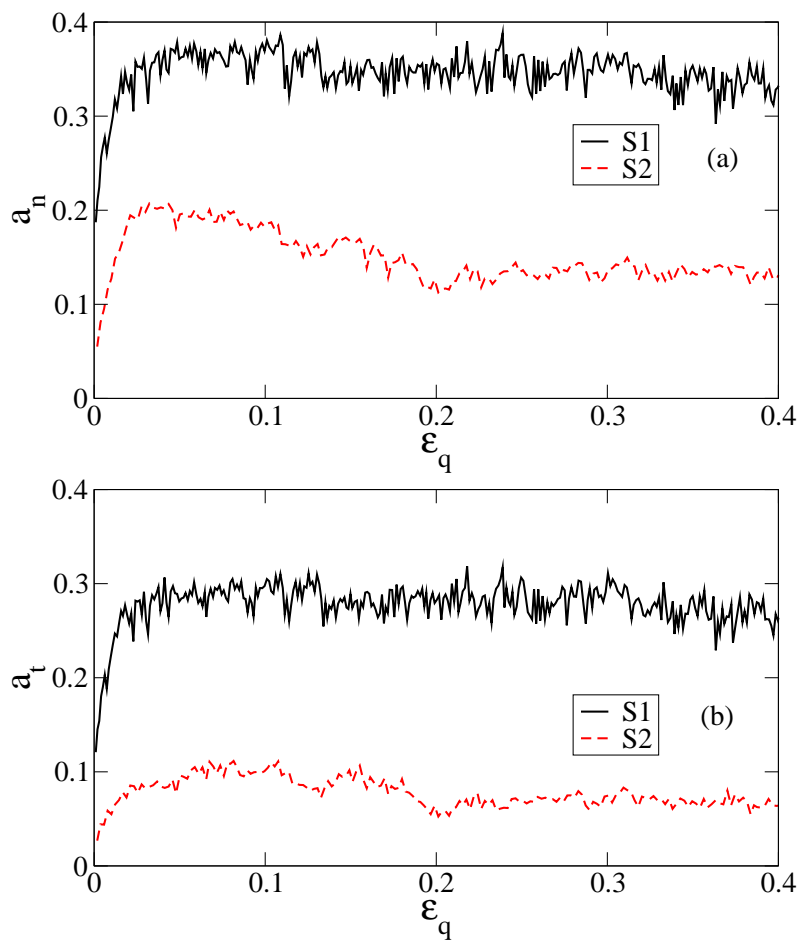

FIG. 11: Evolution of force anisotropies $a_{n}$ (a) and $a_{t}$ (b) as a function of cumulative shear strain $\varepsilon_{q}$ in samples S1 and S2.

Figure 11 shows the evolution of $a_{n}$ and $a_{t}$ with $\varepsilon_{q}$ in samples S1 and S2. We see that, in contrast to fabric anisotropies (Fig. 9), the force anisotropies in pentagon packing remain always above those in the disk packing. This means that the aptitude of the pentagon packing to develop large force anisotropy and strong force chains is not solely dependent on the global fabric anisotropy of the system. In section VI we will show that the force anisotropy of the pentagon packing stems from the high anisotropy of the sub-network of double contacts and strong activation of friction forces. Indeed, due to the geometry of the pentagons, i.e. the absence of parallel sides, the strong force chains are mostly of zig-zag shape, as observed in Fig. 13b, and the stability of such structures requires strong activation of tangential forces. This explains, in turn, the large value of $a_{t}$ for pentagons, very close to $a_{n}$, whereas in the disk packing $a_{t}$ is nearly half of $a_{n}$.

The anisotropies $a, a_{n}$ and $a_{t}$ are interesting descriptors of granular microstructure and force transmission as they underlie the shear stress. Indeed, it can be shown that the general expression of the stress tensor Eq. (5) leads to the following simple relation [13, 39]:

$$
\frac{q}{p} \simeq \frac{1}{2}\left(a+a_{n}+a_{t}\right)
$$

where the cross products between the anisotropies have been neglected and it has been assumed that the stress tensor is coaxial with the fabric tensor Eq. (11) and the 


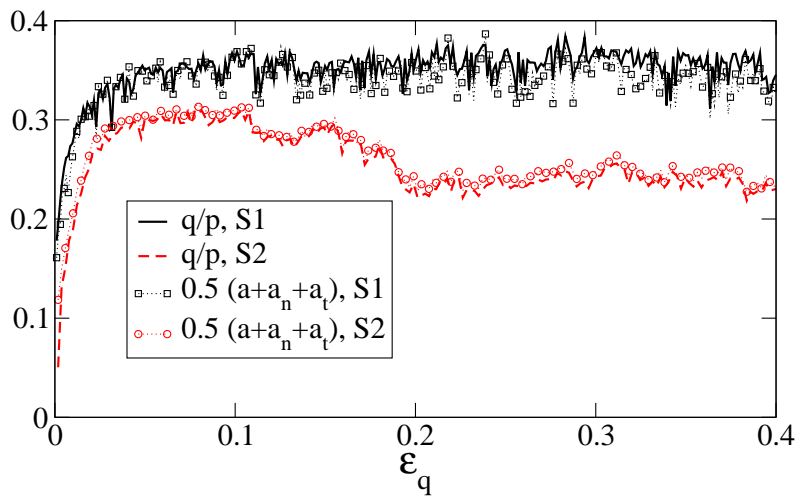

FIG. 12: Evolution of the normalized shear stress $q / p$ for the samples $\mathrm{S} 1$ and $\mathrm{S} 2$ with $\varepsilon_{q}$ together with the corresponding predictions from its expression as a function of fabric and force anisotropies [Eq. (18)].

force tensors Eq. (16). Fig. 12]shows that Eq. (18) holds quite well both for pentagons and disks.

A remarkable consequence of Eq. (18) is to reveal the distinct origins of shear stress in pentagon and disk packings. The fabric anisotropy provides a major contribution to shear stress in the disk packing (Fig. 9) whereas the force anisotropies are more important for shear stress in the pentagon packing (Fig. 11). In this way, in spite of the weak fabric anisotropy $a$, the larger force anisotropies $a_{n}$ and $a_{t}$ allow the pentagon packing to reach higher levels of $q / p$ compared to the disk packing.

\section{B. Force distributions}

The strong inhomogeneity of contact forces is a wellknown feature of granular media. It has been investigated mostly for spherical or cylindrical particles both by experiments and numerical simulations $1,3,3,[4,5,6,6,7,68,9]$. The probability density function (pdf) of normal forces is characterized by two features which seem to be specific to granular media: 1) The pdf is roughly a decreasing exponential function for forces above the mean, 2) In the range of weak forces below the mean, the pdf does not decline to zero with the force. The relative scatter of data reported by different authors for weak forces shows the sensitivity of the pdf in this range to the details of the microstructure. But, the common observation that there is a large number of contacts transmitting very weak forces, is a straightforward signature of the arching effect. From this point of view, one expects that angular particle shape will influence mainly the distribution of weak forces by enhancing the arching effect.

Figure 13 displays maps of normal forces in a portion of each of the samples S1 and S2 at a large cumulative strain. We observe the strong anisotropy of normal forces in the pentagon packing compared to the disk packing (as discussed in section $\mathrm{VD}$ as well as the zig-zag form of the strong force chains. The normal force pdf's are shown in
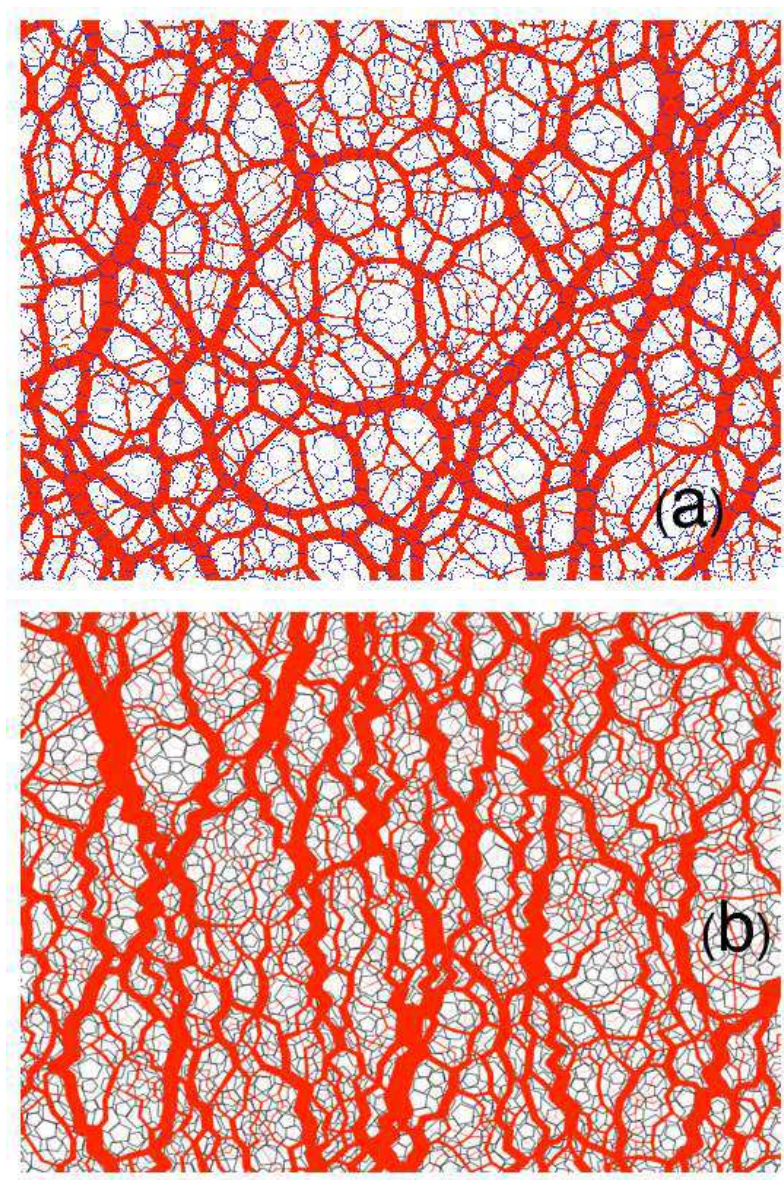

FIG. 13: (color online) Snapshots of normal forces in samples $\mathrm{S} 2$ (a) and S1 (b). Line thickness is proportional to the normal force.

Fig. 14 in log-linear and log-log scales at large strains. The forces are normalized by the mean normal force $\left\langle f_{n}\right\rangle$ in each sample. In both samples, the number of strong forces (above the mean $\left\langle f_{n}\right\rangle$ ) falls off exponentially:

$$
\begin{cases}f_{n} \propto e^{-\alpha_{1} f_{n} /\left\langle f_{n}\right\rangle} & \text { in S1, } \\ f_{n} \propto e^{-\alpha_{2} f_{n} /\left\langle f_{n}\right\rangle} & \text { in S2 }\end{cases}
$$

with $\alpha_{1} \simeq 0.74$ and $\alpha_{2} \simeq 1.4$. The smaller value of $\alpha_{1}$ means that the distribution is wider for pentagons compared to disks. The distribution is nearly uniform in the whole range of weak forces $\left(f_{n}<\left\langle f_{n}\right\rangle\right)$ in S2. In the pentagon packing $\mathrm{S}$, we observe a uniform distribution only in the range $0.1\left\langle f_{n}\right\rangle<f_{n}<\left\langle f_{n}\right\rangle$. Nearly $30 \%$ of forces are in this range. The number of "very weak" forces in $\mathrm{S} 1$ in the range $f_{n}<0.1\left\langle f_{n}\right\rangle$ increases faster than a power law as $f_{n}$ tends to zero. A fraction $\simeq$ $30 \%$ of contacts belong to this range. The presence of numerous "very weak" forces in the pentagon packing is a clear signature of enhanced arching effect that can be characterized, as we shall see below, by the respective roles of simple and double contacts with respect to force transmission. 

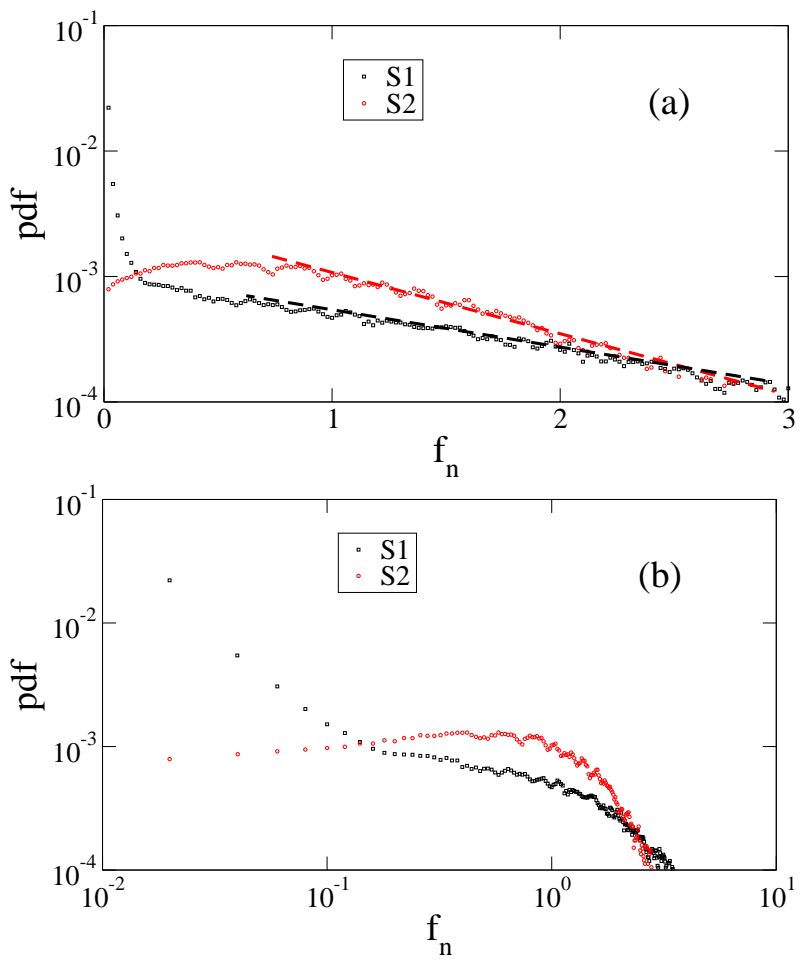

FIG. 14: Probability density functions of normal forces in samples S1 and S2 in log-linear (a) and log-log scales (b).

\section{Bimodal character of stress transmission}

The genuine organization of contact forces in granular media, involving strong force chains propped by weak forces, was first analyzed by Radjai et al. by means of contact dynamics simulations for packings of circular and spherical particles [10]. This analysis proceeds by considering the subset of contacts which carry a force below a cutoff force $\xi$ normalized by the mean force. This subset is referred to as the " $\xi$-network". The variation of a quantity evaluated for the " $\xi$-network" as $\xi$ is varied from 0 to the maximal force in the system, provides its correlation with the contact force. Here, we apply this same approach to $\mathrm{S} 1$ and $\mathrm{S} 2$ samples for the stress ratio $q(\xi) / p$, defined as stress deviator $q(\xi)$ (normalized by the total pressure $p$ of the sample) in the $\xi$-network, and for $a(\xi)$, defined as the fabric anisotropy in the $\xi$-network.

The plot of $q(\xi) / p$ is shown in Fig. 15] for S1 and S2 in the residual state. In both samples, the stress deviator is nearly zero for $\xi<1$, i.e. for the normal forces below the average force. This means that the shear stress is almost totally sustained by the "strong" contact network $\xi>1$ for the pentagon packing as well as for the disk packing. Fig. 16 shows the fabric anisotropy $a^{\prime}(\xi)$ as a function of $\xi$ in the samples S1 and S2. By definition, a positive value of $a^{\prime}$ corresponds to the principal stress direction whereas a negative value corresponds to the orthogonal direction. We see that the direction of anisotropy is orthogonal to the principal stress direction $\left(a^{\prime}<0\right)$ for

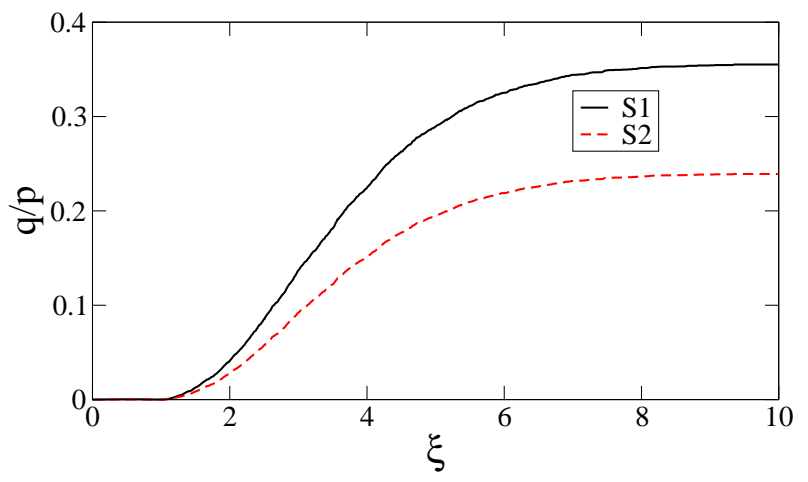

FIG. 15: Partial shear stress $q(\xi) / p$ as a function of force cutoff $\xi$ (normalized by the mean force) for the samples S1 and $\mathrm{S} 2$ in the residual state.

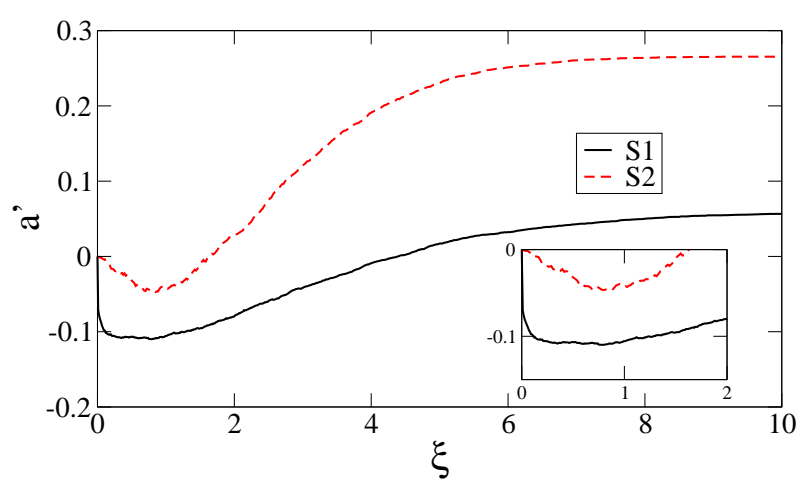

FIG. 16: Partial fabric anisotropy $a^{\prime}(\xi)$ as a function of force cutoff $\xi$ (normalized by the mean force) in the samples S1 and S2.

weak forces (small $\xi$ ). This "orthogonal" anisotropy of the weak forces is more important in the pentagon packing compared to the disk packing, and, as shown in the inset to Fig. 16, it is mainly due to "very weak" forces. When $\xi$ is increased beyond $\left\langle f_{n}\right\rangle, a^{\prime}$ becomes less negative and finally changes sign, showing that the strong contacts are preferentially parallel to the principal axis. These strong contacts are less than $40 \%$ of all contacts, but their positive contribution to $a^{\prime}$ overcompensates the negative contribution weak contacts. For large $\xi$, the partial anisotropy approaches the fabric anisotropy of the whole system.

These data demonstrate the bimodal character of stress transmission also in the pentagon packing in spite of a very different particle geometry. The mean force plays a particular role in differentiating strong contacts from weak contacts. However, the force pdf's (Fig. 14) and the anisotropy of weak forces (Fig. 16) provide also evidence for the existence of a class of very weak forces, corresponding approximately to the range $f_{n}<0.1\left\langle f_{n}\right\rangle$, within the weak network. This class is strongly anisotropic with a privileged direction which is orthogonal to the major principal stress direction, and the corresponding force pdf diverges as the force tends 


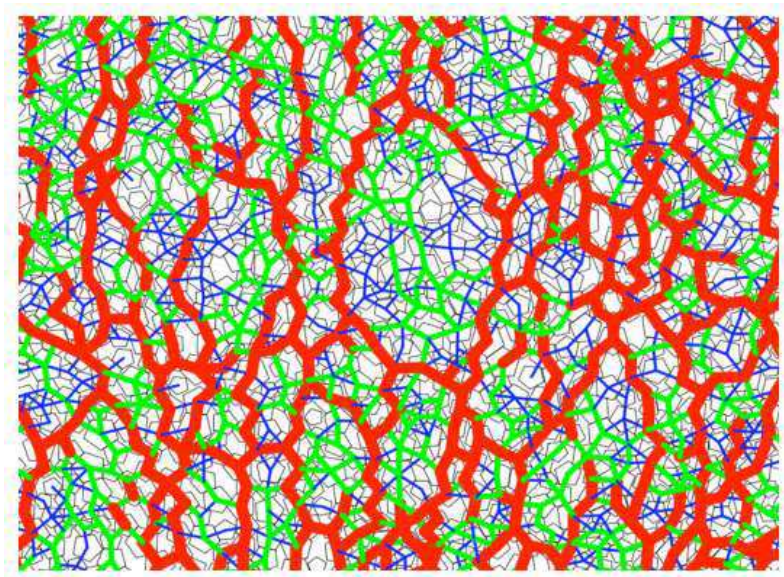

FIG. 17: (color online) Tricolor map of the contact network composed of very weak (blue), intermediate (green) and strong (red) contacts in the pentagon packing.

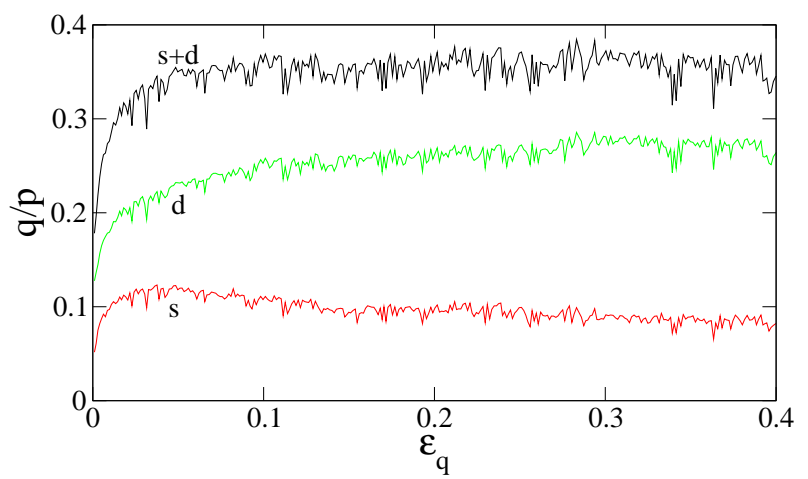

FIG. 18: Normalized shear stress $q / p$ for simple (s) and double (d) contacts, as well as for all contacts $(s+d)$, as a function of cumulative shear strain $\varepsilon_{q}$ in the pentagon packing.

to zero. Fig. 17 displays a tricolor map of the contact network representing very weak, intermediate $\left(0.1\left\langle f_{n}\right\rangle<\right.$ $\left.f_{n}<\left\langle f_{n}\right\rangle\right)$ and strong contacts in the pentagon packing. Large cells of strong contacts are composed of zig-zag chains. The anisotropy of strong contacts is reflected in the elongated shape of these cells along the major principal stress direction. Both intermediate and very weak forces prop these cells.

\section{SIMPLE VERSUS DOUBLE CONTACTS}

In this section, we focus on the organization of simple and double contacts in the pentagon packing. The double contacts, i.e. the side-sharing polygons, are generally assumed to be at the source of the higher strength of polygon packings. For the texture, we would like also to investigate the proportions of simple and double contacts and their respective contributions to the overall anisotropy of the pentagon packing. It is also important to identify the role of double contacts in force transmission.

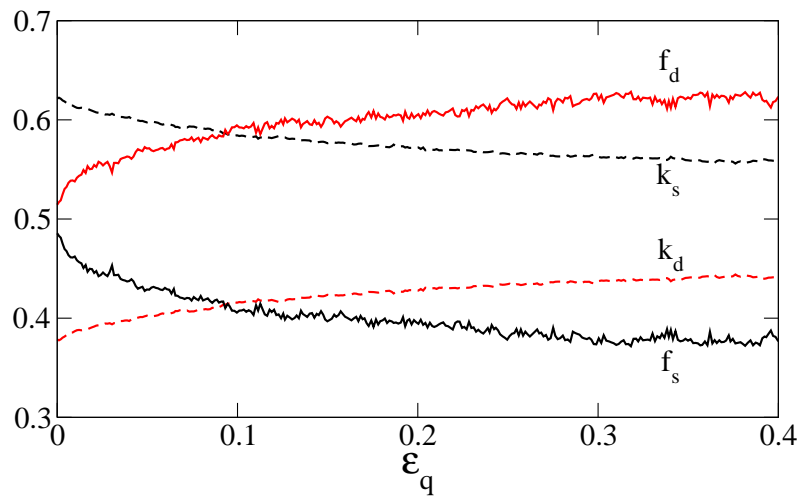

FIG. 19: Proportions $k_{s}$ and $k_{d}$ of simple and double contacts, and the corresponding relative force averages $f_{s}$ and $f_{d}$, as a function of cumulative shear strain $\varepsilon_{q}$.

The general expression [Eq. 5 ] of the stress tensor $\boldsymbol{\sigma}$ allows us to perform a unique additive decomposition of the stress into two parts:

$$
\sigma=\sigma_{s}+\sigma_{d},
$$

where $\boldsymbol{\sigma}_{s}$ is obtained from the expression (5) by restricting the summation to simple contacts, and $\boldsymbol{\sigma}_{d}$ is the complementary tensor involving only double contacts. The respective stress deviators $q_{s}$ and $q_{d}$ normalized by the mean stress $p$ are shown in Fig. 18 as a function of strain $\varepsilon_{q}$. The strength $q_{d} / p$ of double contacts varies from two to three times that of simple contacts during shear deformation of the pentagon packing. The proportions $k_{s}$ and $k_{d}$ of simple and double contacts are shown in Fig. 19 as a function of $\varepsilon_{q}$. The same figure displays the relative force averages $f_{s}=k_{s}\left\langle f_{n}\right\rangle_{d} /\left\langle f_{n}\right\rangle$ and $f_{d}=k_{d}\left\langle f_{n}\right\rangle_{s} /\left\langle f_{n}\right\rangle$, where $\left\langle f_{n}\right\rangle_{s}$ and $\left\langle f_{n}\right\rangle_{d}$ are the mean normal forces of simple and double contacts, respectively. We see that $k_{d}$ increases with strain but remains below $k_{s}$. On the other hand, initially we have $f_{d}=f_{s}=0.5$, reflecting the isotropic state of the packing prepared by isotropic compaction. However, $f_{d}$ increases with shear up to $f_{d} \simeq 1.5 f_{s}$ in the residual state. This means that the larger shear stress carried by double contacts in the residual state is due to the larger mean normal force of double contacts despite their smaller proportion in the packing.

The growth of the number of double contacts shown in Fig. 19 represents the gradual consolidation of the sample. In Fig. 20 we plot the cumulative proportions $\Delta \gamma_{s \rightarrow d}$ and $\Delta \gamma_{d \rightarrow s}$ of simple contacts turning to double and vice versa, respectively. Although transformation between the two contact types occurs at each step in both directions $s \rightarrow d$ and $d \rightarrow s$, the consolidation involves on average a net fraction of simple contacts transforming into double contacts.

The connectivity of the pentagon packing by simple and double contacts can be represented by the proportion $P\left(m_{s}, m_{d}\right)$ of particles with exactly $m_{s}$ simple contacts and $m_{d}$ double contacts. Fig. 21] shows a grey level map 


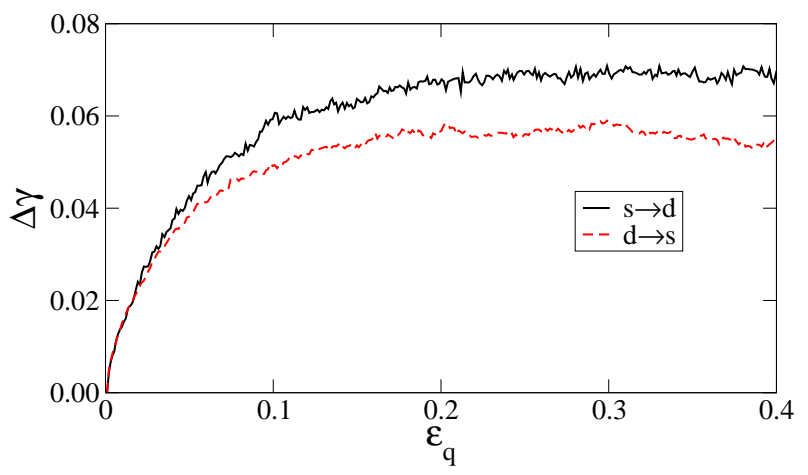

FIG. 20: Cumulative proportion $\Delta \gamma$ of simple contacts turning to double $(s \rightarrow d)$ and vice versa $(d \rightarrow s)$.

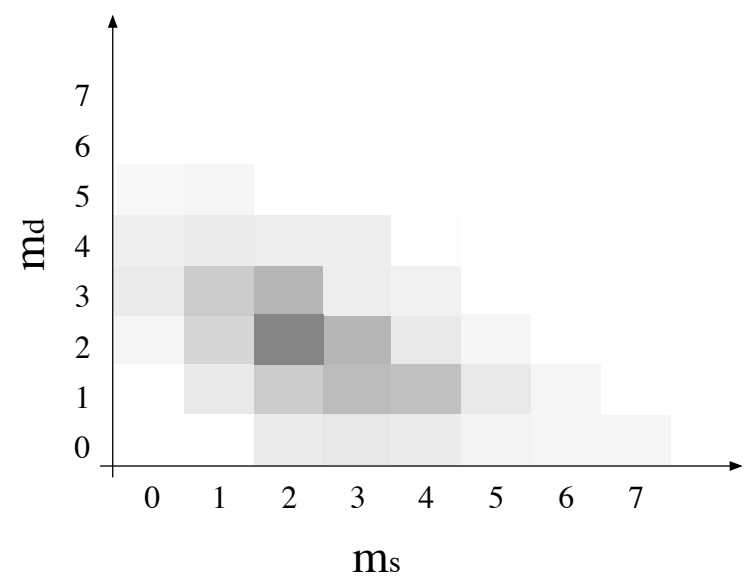

FIG. 21: Grey level map of the connectivity function $P\left(m_{s}, m_{d}\right)$ of the pentagon packing in the residual state.

of this function for the pentagon packing in the residual state. The row $m_{d}=0$ corresponds to particles with only simple contacts (nearly $2 \%$ of the total number of particles) whereas the column $m_{s}=0$ represents the particles with only double contacts (nearly 6\%). On average, a particle has more simple contacts than double contacts but the maximum occurs at $m_{s}=m_{d}=2$.

We now consider the fabric tensor decomposed in a similar way as the stress tensor [Eq. (20)] into two partial tensors:

$$
\boldsymbol{F}=\boldsymbol{F}_{s}+\boldsymbol{F}_{d}
$$

where $\boldsymbol{F}_{s}$ and $\boldsymbol{F}_{d}$ are defined as $\boldsymbol{F}$ in Eq. (11) by simply restricting the summation to simple and double contacts, respectively, and by dividing the sum by the total number $N_{c}$ of contacts. The respective anisotropies $a_{s}^{\prime}$ and $a_{d}^{\prime}$ of simple and double contacts are displayed in Fig. 22 as a function of $\varepsilon_{q}$. The interesting observation here is that the simple contacts have a negative anisotropy which, according to Eq. (13), means that simple contacts are mostly oriented perpendicular to the major principal fabric direction $\theta_{F}$. In other words, most simple contacts belong to the weak network. In contrast, the double

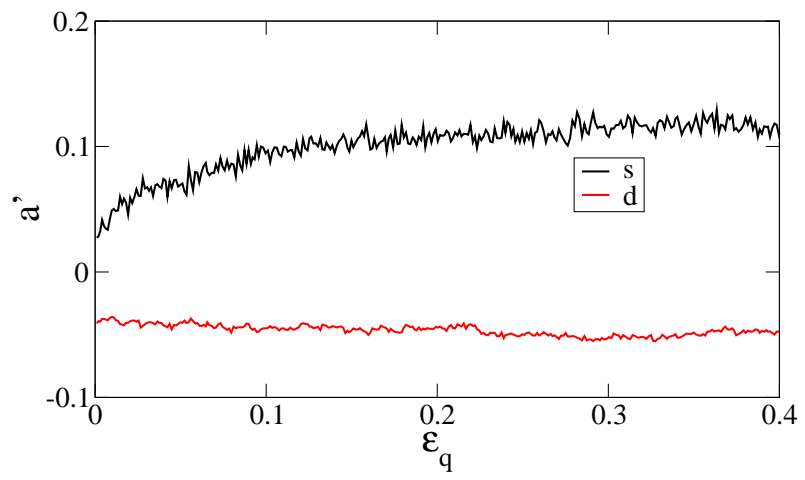

FIG. 22: The anisotropy $a^{\prime}$ of simple (s) and double (d) contacts as a function of cumulative shear strain $\varepsilon_{q}$ in the pentagon packing.

contacts have an increasing positive anisotropy which is larger than the mean anisotropy $a$ of the sample. This is consistent with the fact that the double contacts take over larger forces and they contribute more to the shear stress than simple contacts.

The normal force pdf's for simple and double contacts are shown in Fig. 23. Both contact types are involved in weak and strong networks and the pdf's have the same functional form. But the contribution of simple contacts is more important in the range of very weak forces. Once again, as for anisotropy, the very weak contacts appear to be related to the particular geometry of the pentagons. At large strains, about $32 \%$ of all contacts belong to the very weak force network with $25 \%$ simple contacts against $7 \%$ double contacts. A snapshot of the normal force network is shown in Fig. 24 where the line widths are proportional to the line width with different colors (or grey levels) for simple and double contacts. The remarkable feature of this map is the network of very strong zigzag force chains composed mostly of double contacts and occasionally mediated by simple contacts.

The proportions $k_{s}^{S}$ and $k_{s}^{W}$ of strong (S) and weak (W) simple (s) contacts, respectively, as well as the proportions $k_{d}^{S}$ and $k_{d}^{W}$ of strong and weak double (d) contacts are plotted in Fig. 25] as a function of $\varepsilon_{q}$. We see that in the strong network $\left.\left(f_{n}\right\rangle\langle f\rangle\right)$ the proportion $k_{d}^{S}$ of double contacts is nearly the same as the proportion $k_{s}^{S}$ of simple contacts in the initial (isotropic) state, but during shear $k_{s}^{S}$ declines down to $k_{s}^{S} \simeq 0.5 k_{d}^{S}$ in the residual state, in agreement with the impression left by Fig. 24. We have an inverse situation for the weak network composed of two times more simple contacts than double contacts, i.e. $k_{s}^{W} \simeq 2 k_{d}^{W}$ in the residual state. It is also interesting to remark that the fraction of weak contacts, i.e. $k_{s}^{W}+k_{d}^{W} \simeq 0.58$ in the residual state is very close to that (0.62) in the case of the disk packing. 

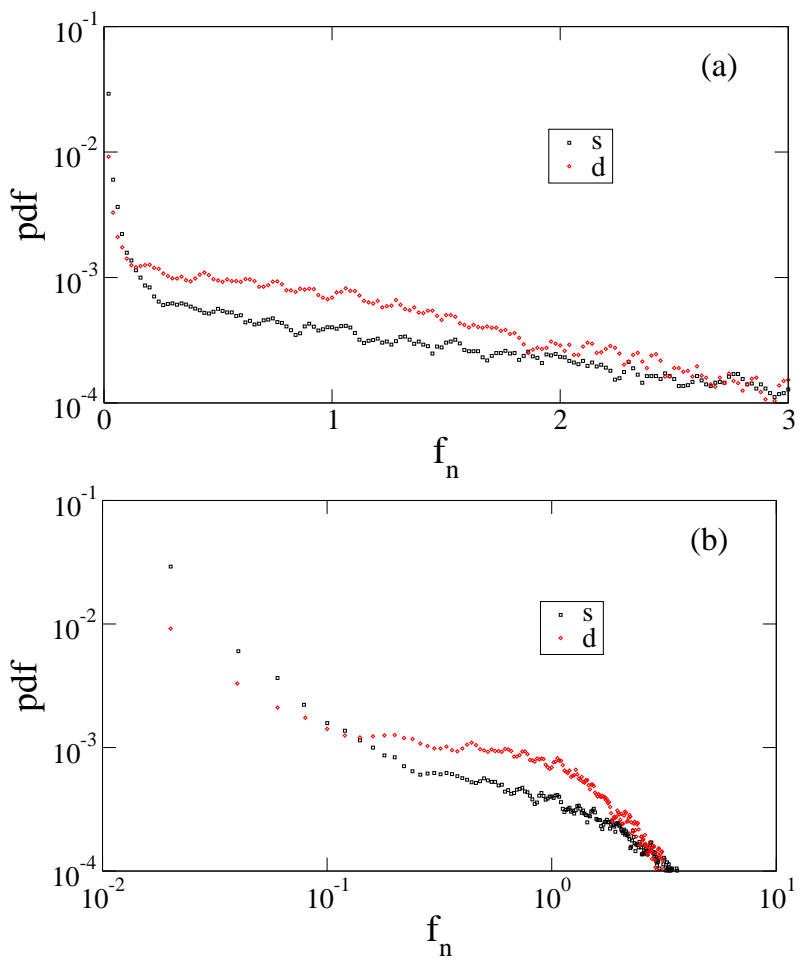

FIG. 23: Probability density function of normal forces for simple (s) and double (d) contacts in log-linear (a) and log$\log$ scales (b).

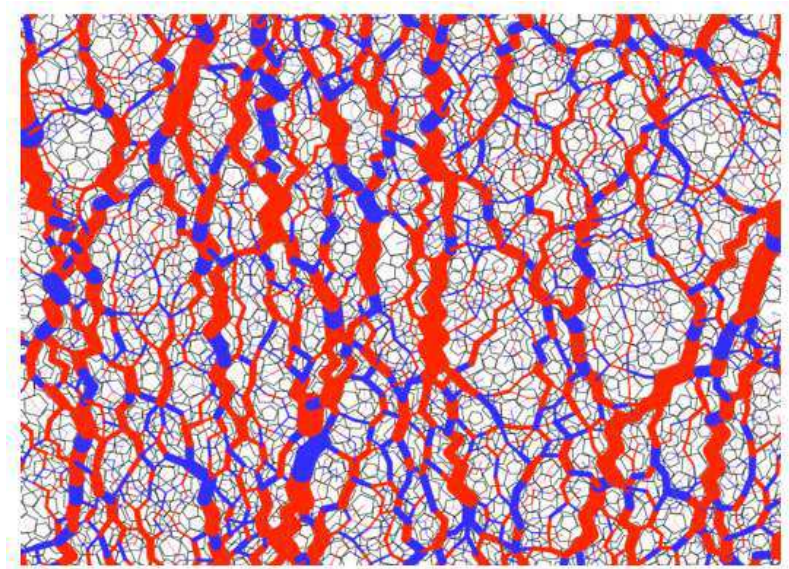

FIG. 24: (color online) Color map of the normal force network in the residual state with simple contacts (s) in blue and double contacts $(\mathrm{d})$ in red. Line thickness is proportional to the normal force.

\section{CONCLUSION}

The objective of this paper was to isolate the effect of particle shape on force transmission in granular media by means a detailed comparison between two similar packings with different particle shapes: pentagons vs. disks. We observed enhanced shear strength and force inhomogeneity in the pentagon packing. But, unexpectedly, the

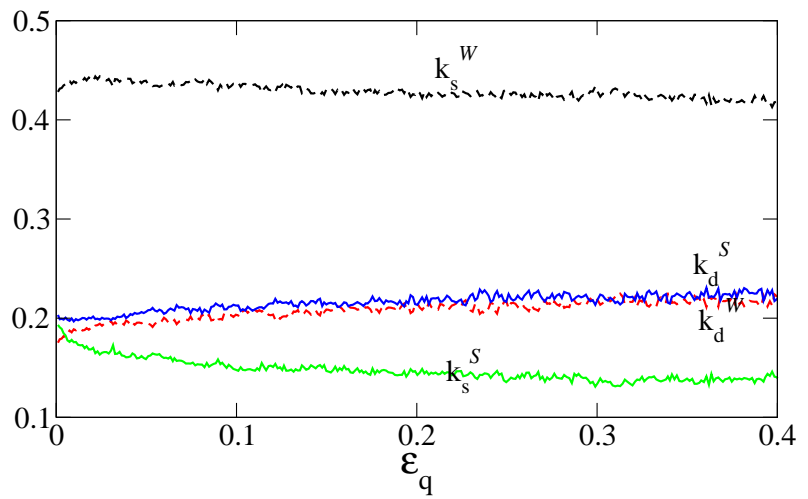

FIG. 25: Proportions $k_{s}^{S}$ and $k_{s}^{W}$ of strong (S) and weak (W) simple (s) contacts, respectively, as well as the proportions $k_{d}^{S}$ and $k_{d}^{W}$ of strong and weak double (d) contacts as a function of cumulative shear strain $\varepsilon_{q}$ in the pentagon packing.

pentagon packing was found to develop a lower structural (fabric) anisotropy compared to the disk packing under shear. This low fabric anisotropy, however, does not prevent the pentagon packing from building up a strong force anisotropy that underlies its enhanced shear strength compared to the disk packing.

This finding is interesting as it shows unambiguously that the force anisotropy in a granular material has two distinct sources: (1) Fabric anisotropy, with a maximum value depending on particle shapes; (2) Particle shapes. The first mechanism is crucial for the disk packing so that the force anisotropy, and the shear stress as a result, vanishes in an isotropic disk packing (e.g. when the friction coefficient is set to zero). The second mechanism may be the predominant source of strength for "facetted" particles that can give rise edge-to-edge (in 2D) or faceto-face (in 3D) contacts allowing for strong force localization along such contacts in the packing. Since the fabric anisotropy is low in a pentagon packing, the role of force anisotropy and thus the local equilibrium structures or arching are important with respect to its overall strength properties. The pentagons analyzed in this work provide thus the first counter-example of a system where the role of fabric anisotropy in shear strength is marginal.

Another shape-related effect was the observation of zigzag force chains mostly composed of edge-to-edge contacts in steady shearing. The vertex-to-edge contacts belong thus mainly to the weak force network or a class of "very weak" forces that can be considered as a signature of enhanced arching or screening effect of forces in the presence of edge-to-edge chains. These "very weak" forces can also be observed, though to a lesser extent, in a disk packing with high coefficients of friction [8] or on experimental pdf's of normal forces acting on the walls of a container [5]. Let us recall that a "very weak phase" was also evidenced by considering the correlation between friction mobilization and the anisotropy of granular texture in a disk packing at the stability limit [14].

By focusing on pentagon packings, we were able 
to demonstrate the nontrivial phenomenology resulting from the specific shape of particles as compared to a disk packing. Although general features of force transmission (pdf's, bimodal character, etc) seem to be robust, the details of force transmission (relative importance of force and fabric anisotropy, the role of edge-to-edge contacts, etc) seem to be strongly shape-dependent. Cur- rently, we work to elucidate this issue for regular polygons (hexagons and higher number of sides) as well as polyhedral particles in three dimensions.

We warmly thank Fréderic Dubois for assistance with the LMGC90 platform used for the simulations. This work was funded by the French Railway Society, the SNCF, and the Région Languedoc-Roussillon of France.
[1] S. J. Antony, Phys Rev E 63, 011302 (2001).

[2] S. N. Coppersmith, C. Liu, S. Majumdar, O. Narayan, and T. A. Witten, Phys. Rev. E 53, 4673 (1996).

[3] C. Liu, S. R. Nagel, D. A. Schecter, S. N. Coppersmith, S. Majumdar, O. Narayan, and T. A. Witten, Science 269, 513 (1995).

[4] T. S. Majmudar and R. P. Behringer, Nature 435, 1079 (2005).

[5] D. M. Mueth, H. M. Jaeger, and S. R. Nagel, Phys. Rev. E. 57, 3164 (1998).

[6] F. Radjai, M. Jean, J. Moreau, and S. Roux, Phys. Rev. Letter 77, 274 (1996).

[7] G. Lovol, K. Maloy, and E. Flekkoy, Phys. Rev. E 60, 5872 (1999).

[8] L. E. Silbert, G. S. Grest, and J. W. Landry, Phys. Rev. E 66, 1 (2002).

[9] S. G. Bardenhagen, J. U. Brackbill, and D. Sulsky, Phys. Rev. E 62, 3882 (2000).

[10] F. Radjai, D. E. Wolf, M. Jean, and J. Moreau, Phys. Rev. Letter 80, 61 (1998).

[11] N. P. Kruyt and L. Rothenburg, ASME Journal of Applied Mechanics 118, 706 (1996).

[12] R. J. Bathurst and L. Rothenburg, J. Appl. Mech. 55, 17 (1988).

[13] L. Rothenburg and R. J. Bathurst, Geotechnique 39, 601 (1989).

[14] L. Staron and F. Radjai, Phys. Rev. E 72, 1 (2005).

[15] H. Ouadfel and L. Rothenburg, Mechanics of Materials 33, 201 (2001).

[16] S. J. Antony and M. R. Kuhn, International Journal of Solids and Structures 41, 5863 (2004).

[17] B. Cambou, P. Dubujet, and C. Nouguier-Lehon, Mechanics of Materials 36, 1185 (2004).

[18] C. Nouguier-Lehon, B. Cambou, and E. Vincens, Int. J. Numer. Anal. Meth. Geomech 27, 1207 (2003).

[19] F. Alonso-Marroquin and H. J. Herrmann, Phys. Rev. E 66, 021301 (2002).

[20] A. Pena, R. Garcia-Rojo, and H. Herrmann, Granular Matter In Press (2006).

[21] G. Saussine, C. Cholet, P. Gautier, F. Dubois, C. Bohatier, and J. Moreau, Comput. Methods Appl. Mech. Eng. 195, 2841 (2006).

[22] J. M. E. Markland, Geotechnique 31, 3,367 (1981).

[23] Wu and Thompson, J Acoust Soc Am 108, 1046 (2000).

[24] J. Mitchell and K. Soga, Fundamentals of Soil Behavior (Wiley, NY, 2005).

[25] M. Jean and J. J. Moreau, in Proceedings of Contact
Mechanics International Symposium (Presses Polytechniques et Universitaires Romandes, Lausanne, Switzerland, 1992), pp. 31-48.

[26] J. Moreau, in Novel approaches in civil engineering, edited by M. Frémond and F. Maceri (Springer-Verlag, 2004), no. 14 in Lecture Notes in Applied and Computational Mechanics, pp. 1-46.

[27] F. Dubois and M. Jean, in Actes du sixième colloque national en calcul des structures - CSMA-AFM-LMS (2003), vol. 1, pp. 111-118.

[28] C. Cholet, G. Saussine, P. Gautier, F. Dubois, C. Bohatier, G. Combe, and K. Sab, in World Congress on Railway Research (WCRR) (2003).

[29] E. Azéma, F. Radjai, R. Peyroux, F. Dubois, and G. Saussine, Phys. Rev. E 74, 031302 (2006).

[30] A. Taboada, K. J. Chang, F. Radjai, and F. Bouchette, Journal Of Geophysical Research 110, 1 (2005).

[31] GDR-MiDi, Eur. Phys. J. E 14, 341 (2004).

[32] J. J. Moreau, in Friction, Arching, Contact Dynamics, edited by D. E. Wolf and P. Grassberger (World Scientific, Singapore, 1997), pp. 233-247.

[33] D. Wood, Soil behaviour and critical state soil mechanics (Cambridge University Press, Cambridge, England, 1990).

[34] F. Radjai and S. Roux, in The Physics of Granular Media, edited by H. Hinrichsen and D. E. Wolf (Wiley-VCH, Weinheim, 2004), pp. 165-186.

[35] A. Taboada, N. Estrada, and F. Radjai, Phys. Rev. Lett. 97, 098302 (2006).

[36] H. Troadec, F. Radjai, S. Roux, and J.-C. Charmet, Phys. Rev. E 66, 041305 (2002).

[37] M. Oda, J. Koshini, and S. Nemat-Nasser, Geotechnique 30, 479 (1980).

[38] B. Cambou, in Powders and Grains 93, edited by C. Thornton (A. A. Balkema, Amsterdam, 1993), pp. 73-86.

[39] F. Radjai, H. Troadec, and S. Roux, in Granular Materials: Fundamentals and Applications, edited by S. Antony, W. Hoyle, and Y. Ding (RS.C, Cambridge, 2004), pp. $157-184$

[40] M. Satake, in Proceedings of the IUTAM symposium on deformation and failure of granular materials, Delft, edited by P. A. Vermeer and H. J. Luger (A. A. Balkema, Amsterdam, 1982), pp. 63-68.

[41] M. Oda and K. Iwashita, eds., Mechanics of Granular Materials (A. A. Balkema, Rotterdam, 1999). 